\title{
DIEZ AÑOS DEL INSTITUTO DE CULTURA MEDITE- RRÁNEA EN LAS ISLAS CHAFARINAS
}

\author{
JUAN ANTONIO BELLVER GARRIDO \\ ANTONIO BRAVO NIETO \\ SONIA GÁMEZ GÓMEZ \\ Instituto de Cultura Mediterránea
}

\section{INTRODUCCIÓN}

El Instituto de Cultura Mediterránea (ICM) es una institución de carácter cultural que nace a mediados del año 2000 estrechamente vinculada al proyecto de investigación sobre las islas Chafarinas. El Instituto se constituye concretamente el 18 de diciembre de 2000, como necesidad de contar con una adecuada estructura de investigación que pudiera hacerse cargo de un ambicioso proyecto cultural en este archipiélago.

Un trabajo publicado hace 56 años por el profesor Carlos Posac Mon (Prehistoria en las islas Chafarinas, 1956) donde se relacionaban algunos hallazgos arqueológicos, fue el revulsivo que despertó la curiosidad hacia las islas por parte de un equipo que quiso documentar la existencia de estos $r$ estos. Ello exigió varios desplazamientos iniciales a las islas, la confirmación de la existencia de objetos de sílex y el novedoso hallazgo de restos de cerámica cardial; los resultados auguraron la transcendencia del que sería el yacimiento más importante del archipiélago, delimitado en el sector sur de la isla del Congeso. Este es el origen de un proyecto cultural que va a estimular la participación de diferentes equipos interdisciplinarios y la colaboración de numerosas instituciones.

El primer viaje ya reveló una complicada tramitación de permisos, gestionados a través del Organismo Autónomo de Parques Nacionales y, posteriormente, autorizados por el Ministerio de Defensa, en cuanto a la estancia,y por el Ministerio de Cultura en lo relativo a la actividad propiamente dicha.También el principio de una forma de trabajar que ha exigido continuos esfuerzos ligados a preparativos logísticos, viajes, coordinación de equipos, gestión administrativa, 
etc. Los sorprendentes resultados obtenidos en las primeras prospecciones motivaron la preparación de la I Campaña Arqueológica en el yacimiento neolítico ya bautizado como Zafrín.

Este fue el principio de una larga serie de trabajos y proyectos vinculados a las islas y, como consecuencia, a la institución gestora del Refugio Nacional de Caza, el Organismo Autónomo de Parques Nacionales (OAPN), que durante los diez años de colaboración y convivencia administrativa apoyó incondicionalmente cada una de las iniciativas propuestas.

\section{LA ARQUEOLOGÍAY LA CREACIÓN DEL ICM}

\section{El yacimiento neolítico de Zafrín}

2001 es el momento en el que se gesta el gupo humano interdisciplinar que acometerá, año tras año, los distintos trabajos. Momentos de compenetración del equipo, unido estrechamente para conseguir llevar a cabo el primer proyecto arqueológico de envergadura en este interesante y novedoso espacio geográfico. Un equipo formado por per sonas vinculadas a la arqueología y a la histor ia, que se organiza inicialmente para una actividad corta aunque intensa, la primera excavación y estudio de las islas. Se trató de una exper iencia de quince días en la que diferentes personas formadas en varias disciplinas tendrían que convivir junto a un equipo de guardas y biólogos que promueven sus estudios en la Estación Biológica de las islas Chafarinas. Pero el factor que infunde más complicación y más noredad al proyecto vino determinado por el peculiar emplazamiento del yacimiento en la isla del Congreso, espacio que ha pemanecido deshabitado prácticamente desde el Neolítico hasta la actualidad, y que flanquea el margen occidental del arhipiélago. El desplazamiento en zodiac al y acimiento varias veces al día, transportando al personal junto a todos los utensilios necesar ios, se antojaba como un auténtico acontecimiento, no exento de dificultades e incertidumbre.Y ello porque el trabajo diario dependía, entre otras muchas cosas, de que el estado del mar permitiese poder desembarcar o no. El éxito de este primer ensayo afianzó al ICM en las islas Chafarinas, fortaleció la cohesión de su equipo y, en virtud del trabajo realizado, consolidó su propuesta de investigación ante las autoridades competentes. De esta forma, y año tras año, lo que fue no vedoso ha pasado a con vertirse hoy en un trabajo cercano, sentido como algo propio. 


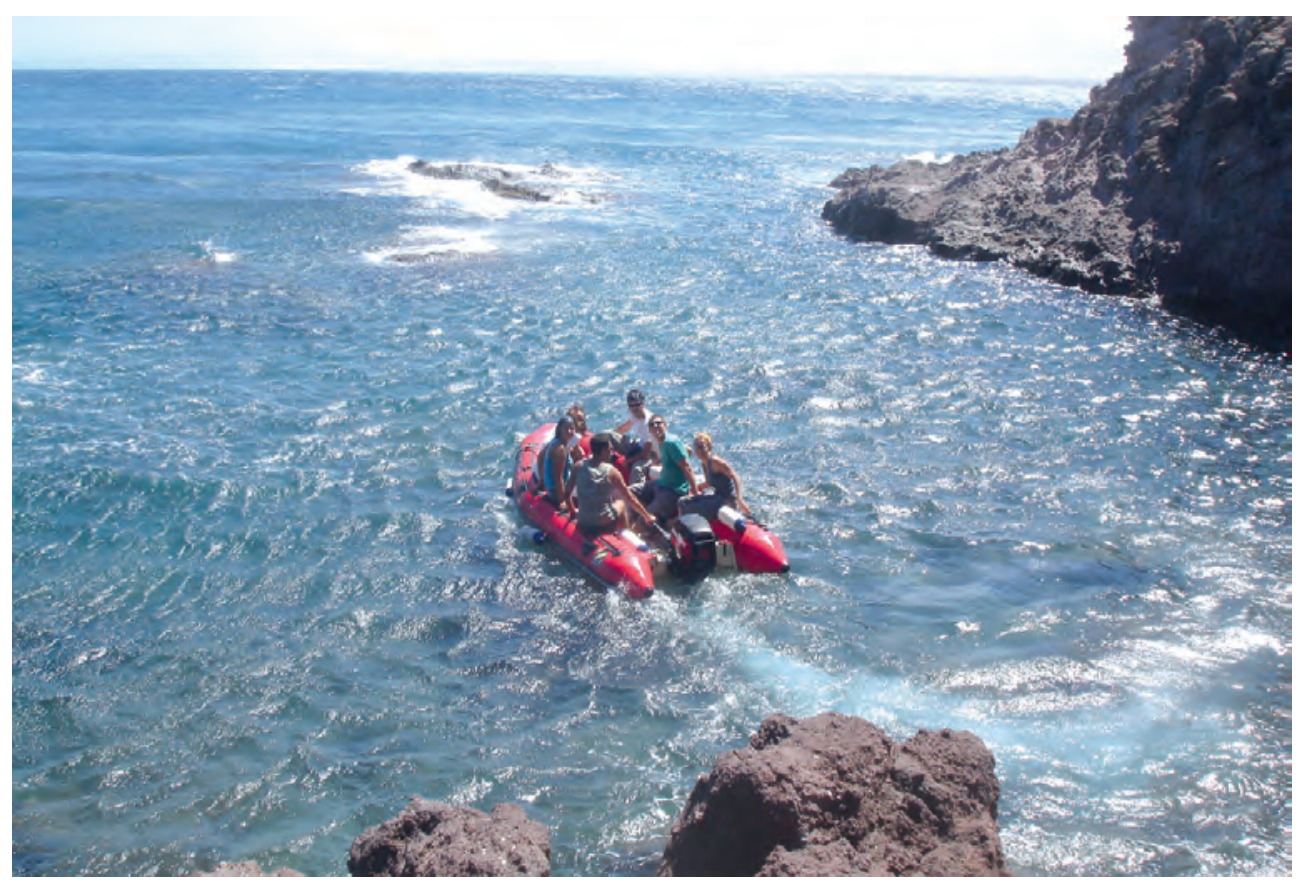

Evacuación del equipo de arqueología en zodiac desde la isla del Congreso a la de Isabel II cuando comenzaba a entrar un temporal de poniente.

La continuación del proyecto vino determinada por el éxito de esta pr imera excavación, pues confirmó la existencia de un importante lugar de hábitat del Neolítico antiguo cardial, con la existencia de estructuras domésticas (hogares, cubetas, etc.) y una asombr osa riqueza de materiales arqueológicos. Todo esto permitió establecer planteamientos futuros con sólidas aspiraciones científicas e ir perfilando la configuración de un equipo profesional. A su vez, comenzaron a surgir proyectos paralelos relacionados con el medioambiente, orientados a prestar apoyo a las venideras expediciones arqueológicas y que fueron sufragados y patrocinados por Parques Nacionales, la Consejería de Cultura de la ciudad autónoma de Melilla y la Fundación GASELEC.

Pasaron los años entre diferentes intervenciones arqueológicas en Zafrín. De todas ellas habría que destacar cuatro grandes campañas, no las únicas, pero sí fueron las de mayor envergadura la de los años 2001,2003, 2004 y 2005. Este penúltimo año fue clave por los prometedores resultados obtenidos, momento en que el ICM firmaba un convenio con la Universidad de Valladolid y se ponía en marcha una larga campaña de 45 días en las islas y un amplio despliegue de un 
equipo compuesto por unas 18 personas, muy superior al de cualquier otro proyecto anterior llevado a cabo en las islas.

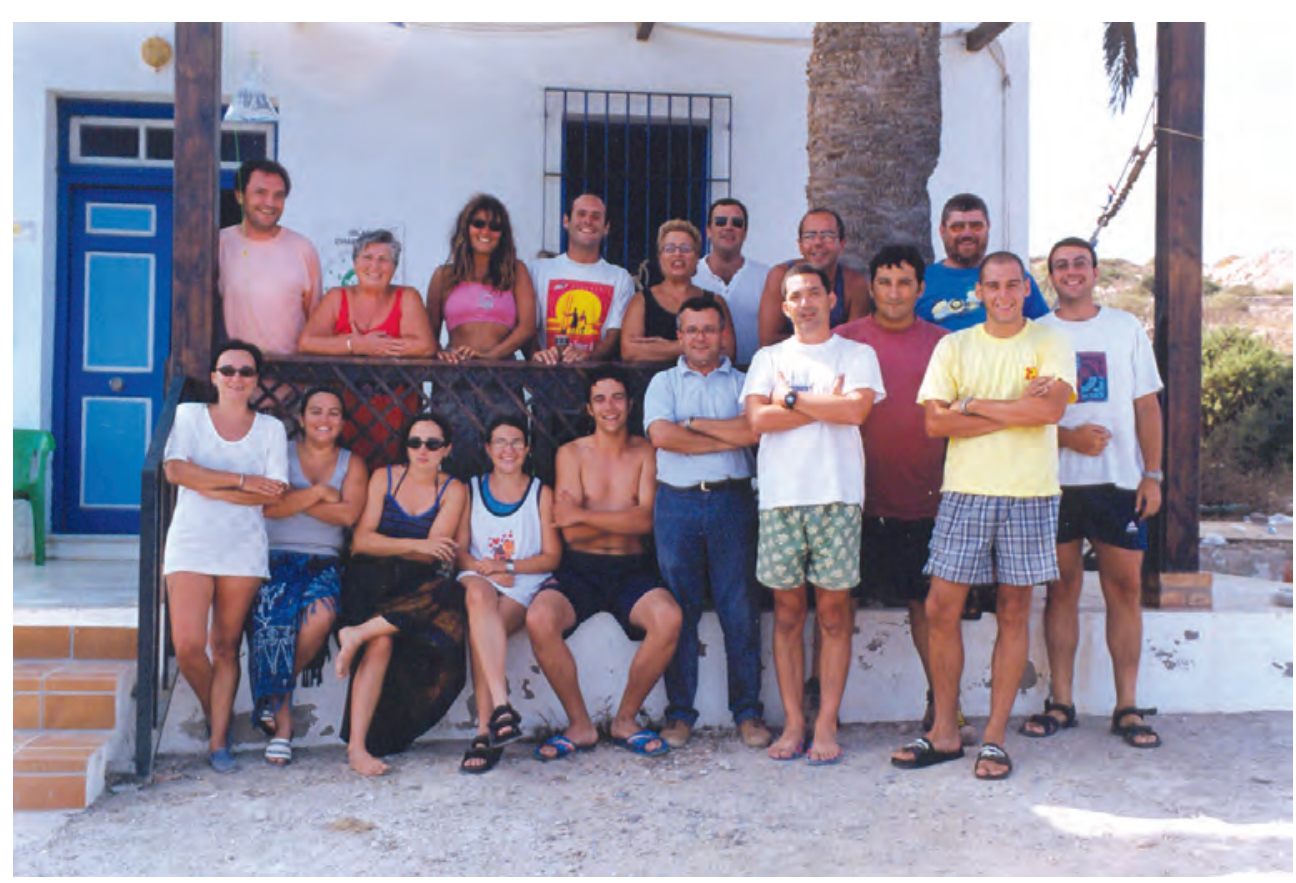

Equipo de excavación en la primera campaña, año 2001.

Los resultados de la campaña de 2004 superar on las expectativas que a priori se esperaban de la potencialidad del ycimiento. En esta campaña se trabajó en una extensa ár ea de excavación que permitió documentar el perímetr o completo de una interesantísima estructura de hábitat que resultaba excepcional dentro del panorama del Neolítico Antiguo norteafricano. Este año se localizó una cabaña completa y v arias estructuras exteriores relacionadas con ella. De esta campaña se extrajer on numerosas y div ersas muestras que ser virían para realizar varios análisis paleoeconómicos y paleoambientales que pr oporcionarían datos de un importante valor. Sin embargo, durante la intervención también sería constatada la intensa er osión que sufría el y acimiento, provocada por su ubicación en una pendiente muy acusada y que lo está destruyendo por arrastre. 


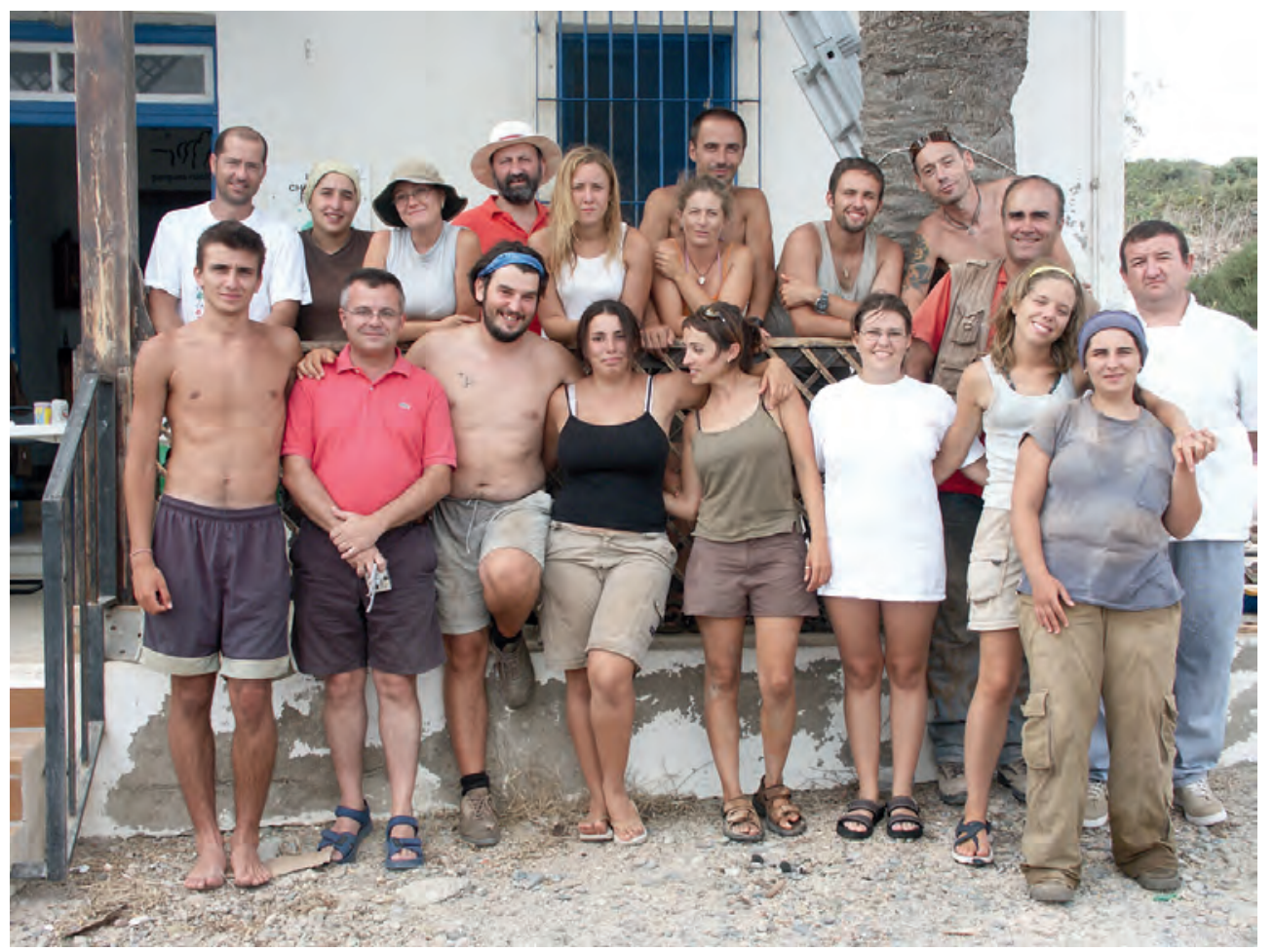

Equipo de excavación en la campaña de 2004.

\section{Prospecciones arqueológicas en la isla del Rey y excavación del yacimiento de La Plataforma en la isla del Congreso}

Durante estos años, la gestión arqueológica no estuvo centrada únicamente en el yacimiento neolítico de Zafrín, sino que el ICM realizó desde sus primeras visitas al archipiélago diferentes intervenciones paralelas, sobre todo en la isla del Rey y del Congreso. Las tres islas fueron prospectadas en diferentes ocasiones por varios equipos organizados para realizar barridos sistemáticos en superficie que sirvieron para acotar los límites del yacimiento neolítico y localizar nuevos espacios de interés arqueológico.

El proyecto de intervención en la isla del Rey tuvo como objetivo ampliar los conocimientos sobre el poblamiento prehistórico de las islas Chafainas, complementando la información que ya aportaba el yacimiento de Zafrín en la isla del Congreso. Por lo tanto, existía la necesidad de estudiar de foma conjunta los testimonios documentados en ambas islas, dada la conocida existencia de vetas 
de sílex rojo y amarillo en la del Rey. La metodología aplicada en la prospección arqueológica en esta isla se basó en el Sistema de Regsstro Temático y Espacial de Yacimientos en Superficie (SIRTEYS). Las características metodológicas de este sistema permitían aunar perfectamente los objetivos de investigación arqueológica y de protección patrimonial, tanto cultural como ambiental, que se pretendían alcanzar. Esta metodología estaba basada en la utilización de æceptores GPS para cuyo correcto funcionamiento eran necesarias una serie de características geográficas y ambientales que se cumplían perfectamente en esta isla. El equipo de prospección efectuó una exploración exhaustiva de la superficie en busca de materiales arqueológicos o de indicios visibles de estructuras, tarea que se desarrolló durante la campaña arqueológica de 2005.

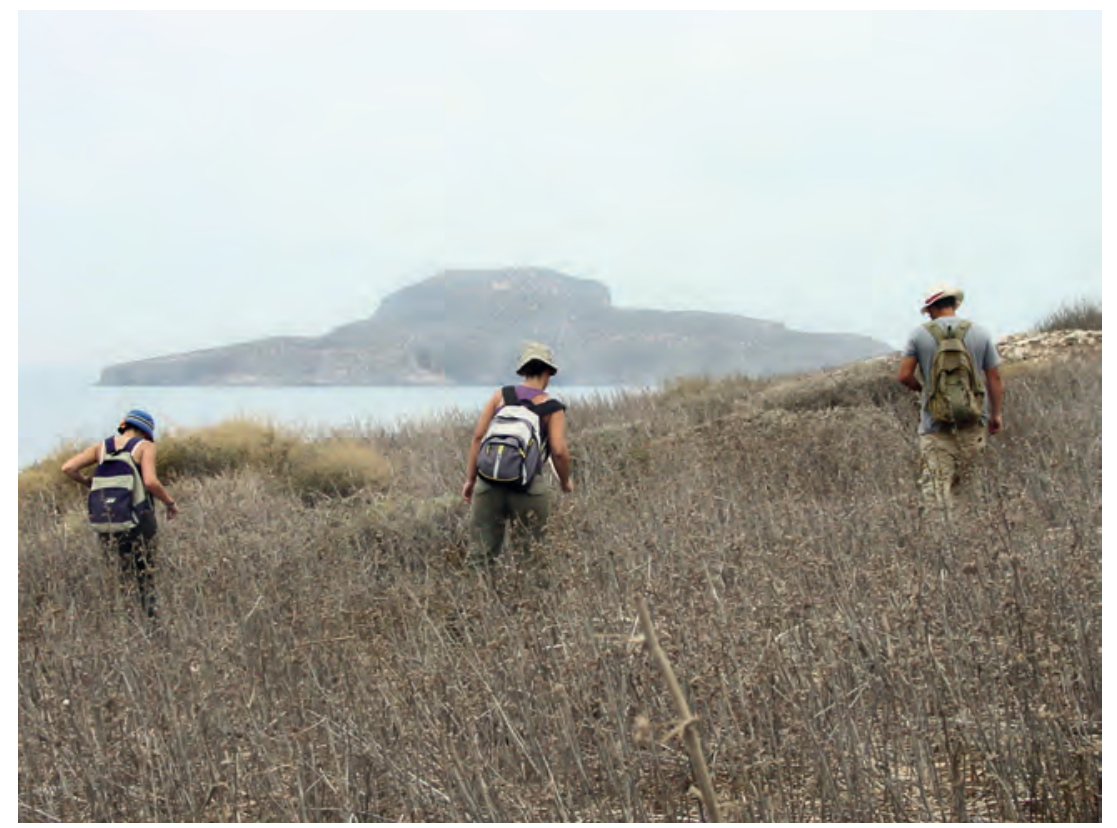

Arqueólogos del Instituto de Cultura Mediterránea durante las prospecciones en la isla del Rey en el año 2005.

Los resultados de las prospecciones intensivas en el año 2001 en la isla del Congreso precisaron la delimitación del y acimiento neolítico e incor poraron nuevos descubrimientos arqueológicos. Lo más significativo fue desvelar la existencia de una serie de aterrazamientos o bancales de piedra en el sector nowriental 
de la isla, o la presencia de una construcción defensiva en la parte más occidental, en el sector sur. El espacio ocupado por estos bancales, alrededor de una hectárea, iba a constituir una interrogante para el equipo de investigación, pues el hecho de no tener conocimiento de una ocupación humana posterior a época neolítica llevó a los investigadores a sospechar que esta construcción podría tener una cronología antigua y asociada al y acimiento prehistórico. Finalmente, durante la campaña de 2005 se r ealizó una inter vención que dataría los aterrazamientos en época moderna.

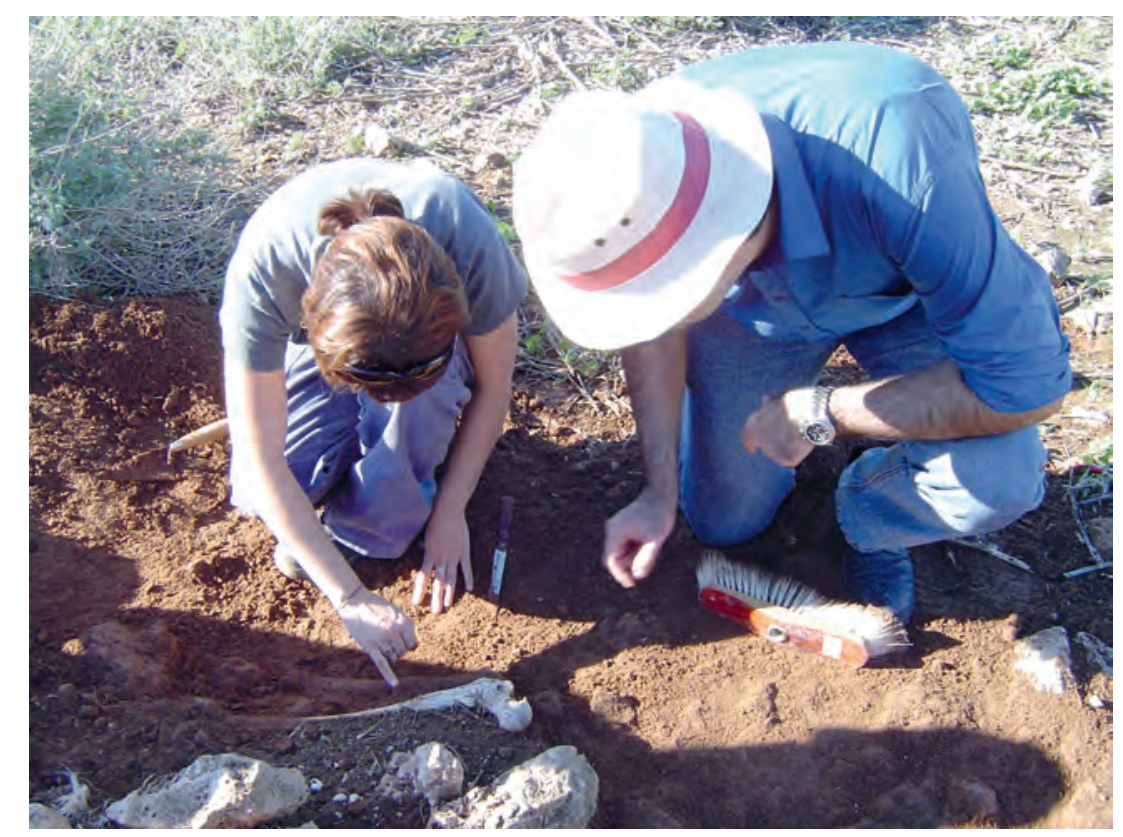

Intervención en la isla del Rey ante el descubrimiento de restos humanos durante las primeras prospecciones en diciembre de 2003.

Entre los años 2006 y 2007 se excró el yacimiento de época contemporánea La Plataforma, situado en posición elevada en la parte sureste de la isla del Congreso, ladera oriental de la misma. Se trata de un edificio de foma rectangular que albergaba a un contingente militar reducido, destinado en la isla con un fin defensivo. Un for tín o cuartel de vigilancia de aproximadamente $100 \mathrm{~m}^{2}$, construido poco después de la toma de las islas, entre finales del siglo XIX y principios del XX. La campaña de 2006 no fue suficiente para exca var todo el 
edificio y no se concluyó el proyecto hasta la intervención en el verano de 2007. Los resultados de ambas exca vaciones llevaron a relacionar directamente este edificio con los aterrazamientos construidos en la zona norte de la isla, interpretados como posibles bancales destinados al cultiv o y probablemente al autoabastecimiento de este contingente. Las prospecciones realizadas en campañas anteriores en este sector aterrazado de la isla, concluyeron que el material arqueológico que aparecía en su entorno era de cronología moderna y que por tanto dichas construcciones no tenían ningún vínculo con el yacimiento prehistórico de Zafrín. Además, la constitución de los m uros del edificio y la de las terrazas mostraron idéntica factura, lo que reafirmó la idea de que se llevaron a cabo en un mismo momento, durante la ejecución de un proyecto defensivo para la isla del Congreso.

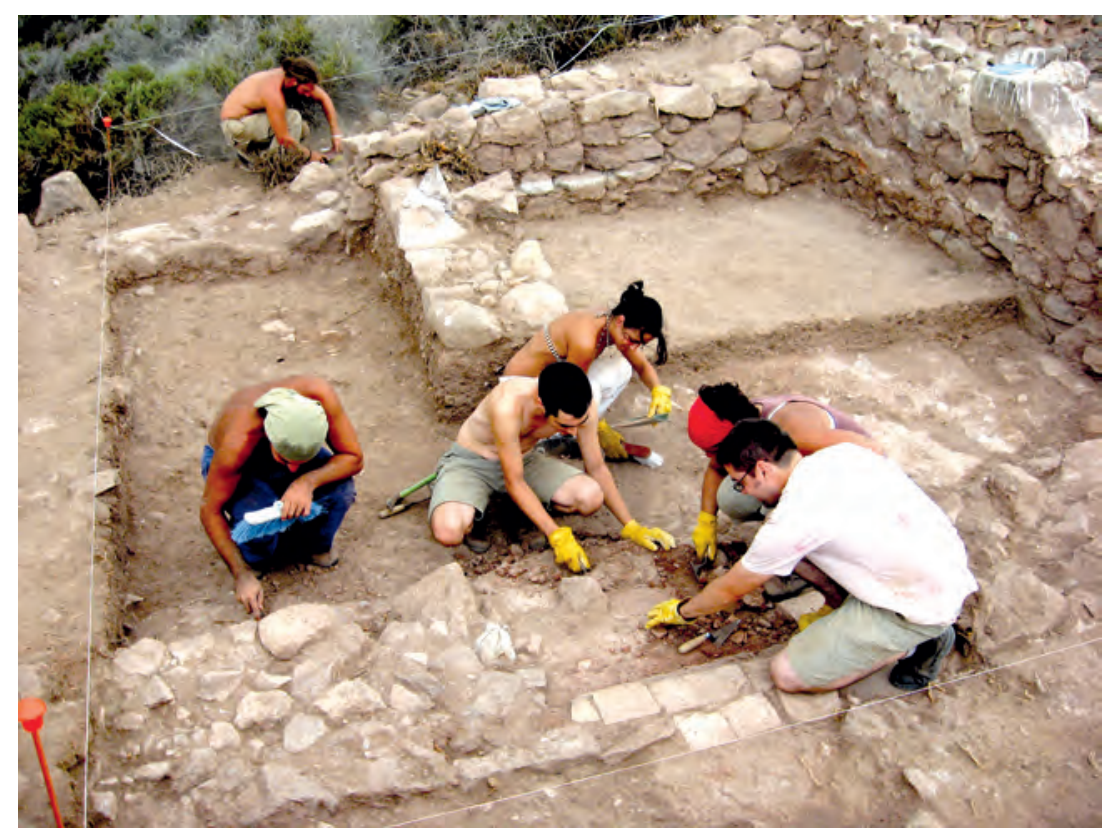

Excavaciones en el yacimiento de La Plataforma en la isla del Congreso con estudiantes de la Universidad de Málaga.

Desde los primeros años arqueológ icos en las Chafar inas, el Instituto Geológico Minero trabajó junto al ICM en un estudio geológ ico de las islas y, concretamente, en la lóg ica y sugestiva hipótesis surgida de que éstas estaban unidas a la costa y formaban un cabo o promontorio estratégico en época neolítica. 
Presunción que surge al pensar en la gran dificultad que sería habitar en las islas para aquellos pobladores neolíticos, no solo por el espacio reducido en el que se encontraban, sino porque sería necesario transportar el agua potable y las piezas de caza cobradas por medios marítimos hasta las mismas. Los resultados fueron de gran interés para conocer el desarrollo del yacimiento y las circunstancias que llevaron a su abandono.

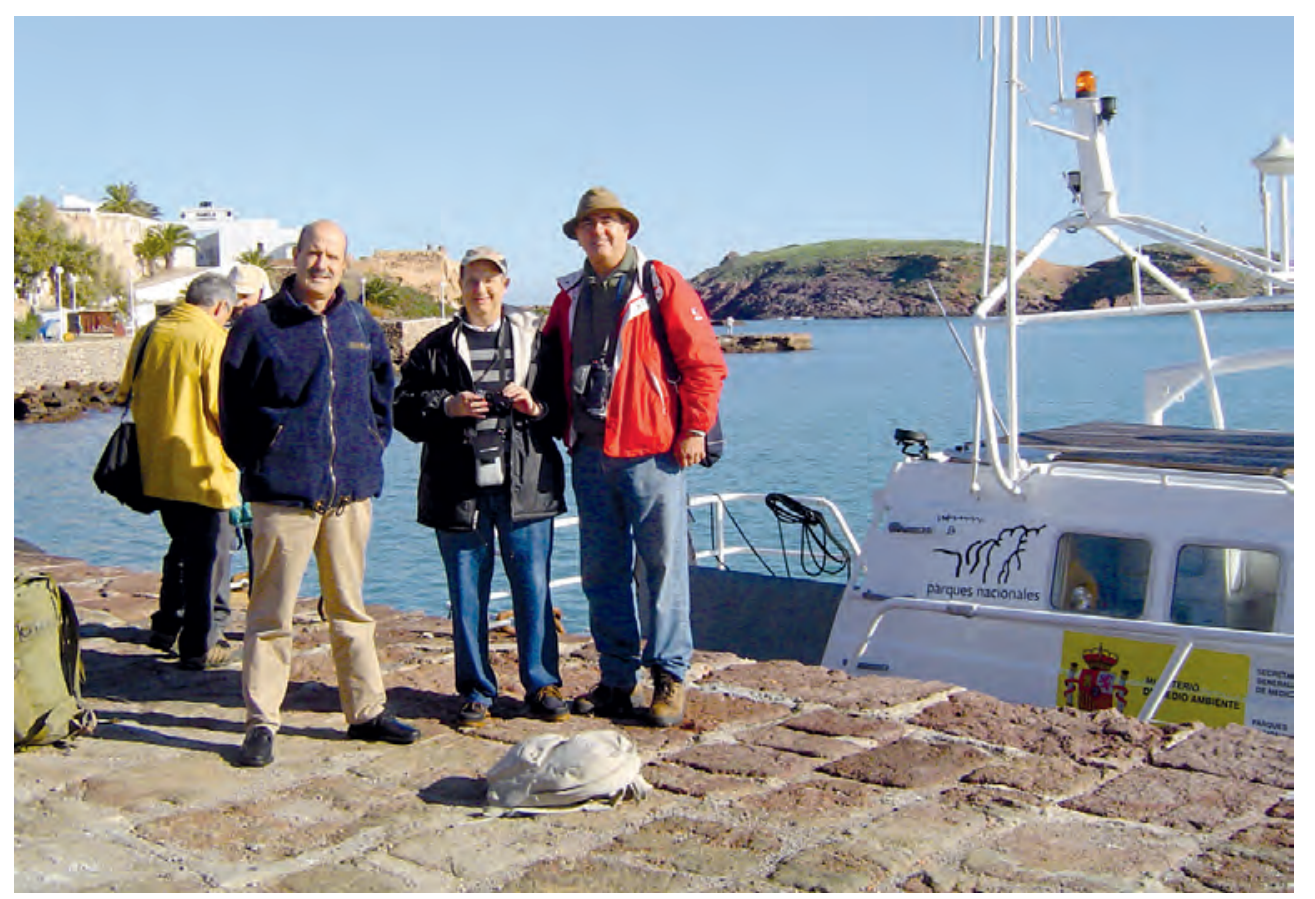

Llegada al puerto de Chafarinas del grupo de geólogos que participaron en el proyecto del yacimiento de Zafrín.

\section{El estudio de impacto ambiental de las intervenciones arqueológicas}

El Proyecto Chafarinas tenía como uno de sus objetivos el estudio y excavación del conjunto de y acimientos arqueológicos situados en las islas del Re y y especialmente en la del Congreso. La superficie afectada por estas actividades de investigación en este enclave protegido iba a ser de cier ta importancia, por lo que se hizo necesario estudiar una serie de medidas correctoras de carácter ambiental para evitar su alteración. Se acometió por esta razón la $r$ egulación de todas las actividades, tanto extractivas, propias de las excavaciones, como otras 
de carácter prospectivo, que se extienden por todo el encla ve insular. El status de área protegida motivó la necesidad de actuar en ese sentido y morió al ICM a presentar a la Fundación Biodiv ersidad un proyecto de evaluación de impacto denominado Restauración de hábitats naturales sometidos a intervenciones arqueológicas en el Refugio Nacional de islas Chaf arinas, dependiente del minister io de Medio Ambiente. Las futuras actividades arqueológ icas debieron seguir convenientemente la regulación que surgió de aquel trabajo a fin de $\mathrm{r}$ espetar el entor no desde un punto de vista medioambiental a la ez que se desarrollaban los trabajos extractivos. En este sentido finalizar on, dentro del Plan Nacional de Acción de Voluntariado del Organismo Autónomo de Parques Nacionales en el verano de 2004, las obras de construcción de un vivero para la reproducción de especies vegetales autóctonas, un complejo de más de $200 \mathrm{~m}^{2}$, en continuo crecimiento y perfeccionamiento, que permitiría la repoblación adecuada de cualquier sector necesitado de las islas, pero construido principalmente como complemento de las actividades arqueológicas.

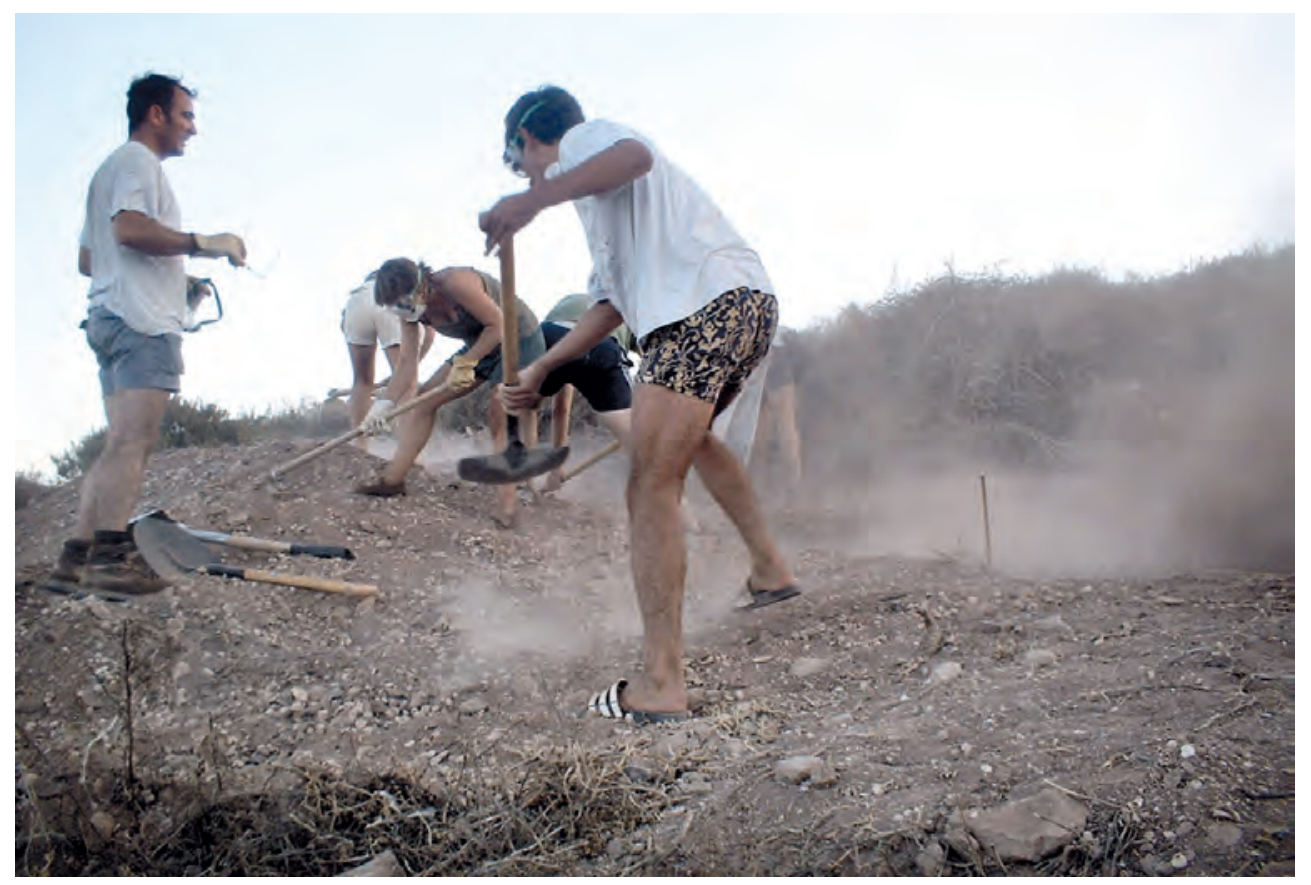

Trabajos para volver a cubrir la superficie excavada durante las excavaciones de 2003. Aplicación de las medidas aportadas por el estudio de Impacto Ambiental. 
En aquel trabajo se pretendía equilibrar y compensar las actividades científicas que vinculan la protección del patrimonio histórico y cultural con las de patrimonio medioambiental que están establecidas para el Refugio Nacional de Caza de islas Chafarinas.

\section{NUEVOS PROYECTOS, PROPUESTAS Y CAMPOS DE VOLUNTARIADO DEL ORGANISMO AUTÓNOMO DE PARQUES NACIONALES}

Digitalización de la evolución constructiva en las islas Chafarinas desde 1848

Este proyecto fue iniciado en 2007 y lle vado a cabo por el ICM para el Organismo Autónomo de Parques Nacionales. Se denominó Catalogación, clasificación y digitalización de expedientes de obras públicas y privadas sitos en los archivos nacionales. En el verano de 2009, después de analizada gran parte de la cartografía referente a las obras de construcción de las islas Chafarinas, se llevó a cabo un trabajo basado principalmente en localizar y topografiar los restos de los edificios más impor tantes ya desaparecidos. Estas evidencias iban a formar parte de un plano integral de las construcciones del archipiélago, con los edificios más destacados, principalmente en la isla de Isabel II a lo largo de su historia.

El proyecto quedó dividido en dos fases claramente diferenciadas y complementarias entre sí. En primer lugar y, con el fin de ofrecer una visión conjunta del entramado urbanístico, se realizó una recopilación y clasificación de toda la documentación (textual y g ráfica) relacionada con las obras de construcción proyectadas en las islas Chafarinas desde su ocupación en 1848 hasta nuestros días. El desarrollo de la investigación tuvo lugar en diferentes archivos nacionales: Archivo Intermedio Militar de Melilla; Autoridad Portuaria de Melilla; Archivo General Militar (Instituto de Histor ia y Cultura Militar) en Madrid; Cartoteca Histórica del Servicio Geográfico del Ejército (Madrid) y Archivo General Militar de Segovia. También se consultaron fondos privados de difícil acceso, debido a su carácter no púb lico, como varias colecciones particulares.

La segunda fase del proyecto consistió en el estudio de la documentación obtenida en cada uno de los ardivos y en la localización, a través de la cartografía histórica, de todas las edificaciones desaparecidas. El objetivo fundamental de 
la última etapa del proyecto fue aportar todos los datos e información posibles sobre la ubicación de cada una de las constr ucciones, tanto si existían en la actualidad como si no. Se llevó a cabo un trabajo topográfico que recogería todos los restos constructivos dispersos en el terreno perteneciente a los edificios desaparecidos. Junto con las construcciones existentes y en uso, estos edificios olvidados formarían parte de un plano integral, asociado a sus tablas de atributos y datos espaciales, mostrando la fisonomía que un día tuvieon las islas. Estos datos acerca de la evolución aparente del archipiélago servirían para realizar un documento completo con infor mación pormenorizada de todos los edificios y usos de los mismos a lo largo de la histor ia de las islas que a yudarían a entender su evolución desde los pr imeros edificios constr uidos a mediados del siglo XIX hasta la actualidad. La digitalización de la evolución de las infraestr ucturas defensivas, de los edificios militares y particulares, los puertos o aljibes mostró el esplendor urbano que tuvo la isla Isabel II, la única habitada en permanencia y la que ha sufrido la mayor presión humana desde su ocupación.

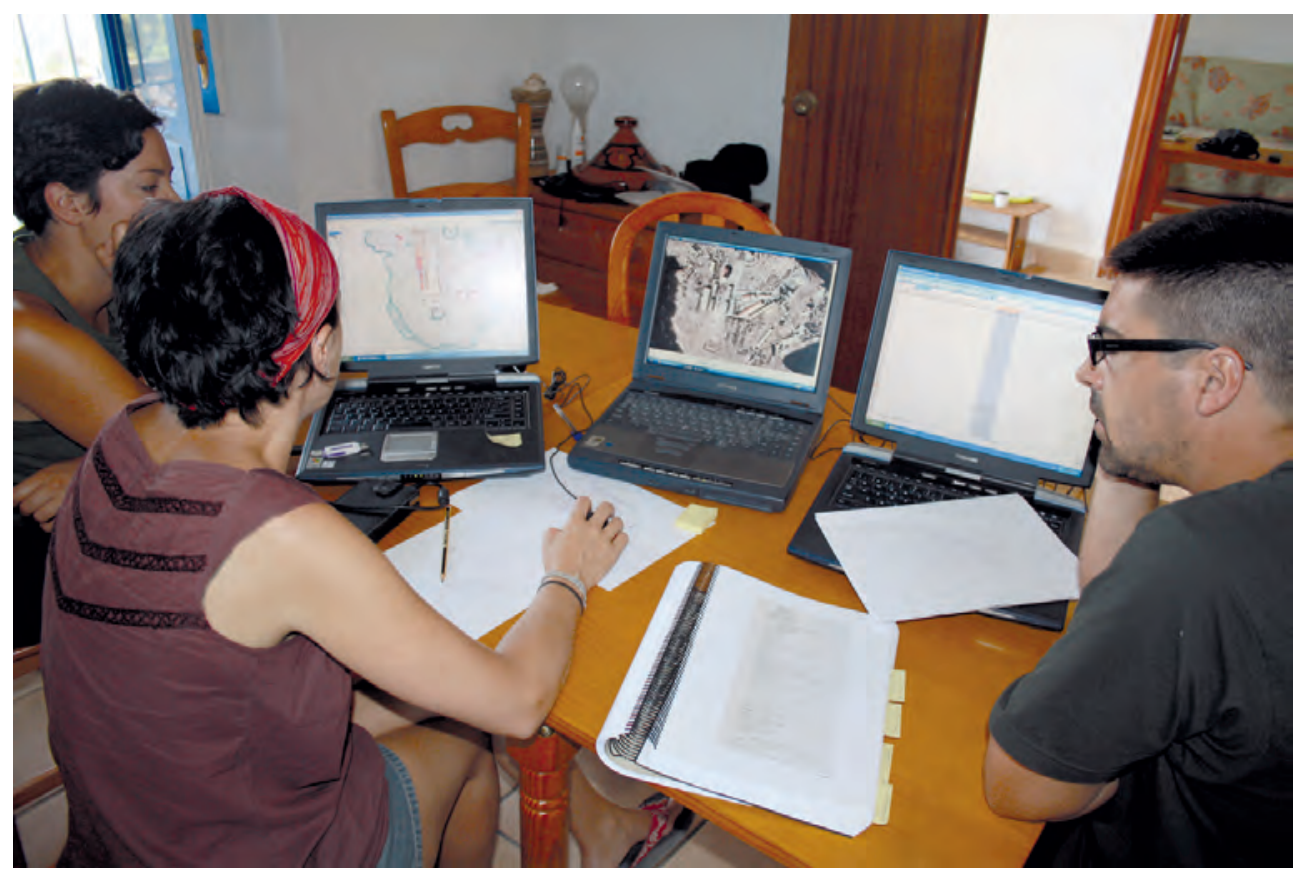

Equipo de trabajo en las islas durante la realización del proyecto "Catalogación, clasificación y digitalización de expedientes de obras públicas y privadas sitos en los archivos nacionales”. 


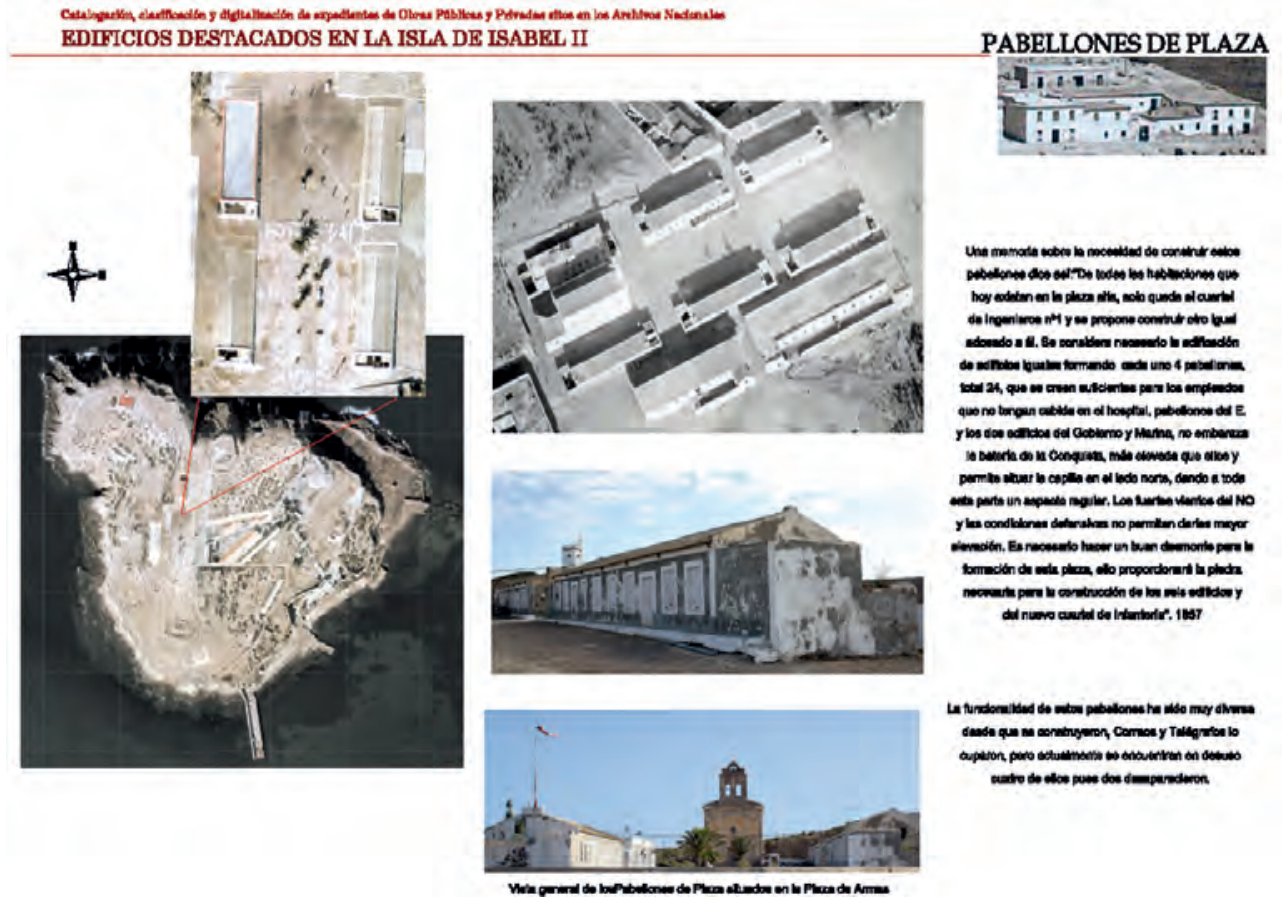

El proyecto aportó importantes datos acerca de la evolución constructiva en las islas Chafarinas desde su ocupación en 1848 .

\section{El Centro de Interpretación de islas Chafarinas}

Entre las iniciativas que se llevaron a cabo en las islas habría que destacar una propuesta que no llegó a ma terializarse pero que quedó planteada para un posible desarrollo posterior. Para el año 2002 el ICM pr opuso al Organismo Autónomo de Parques Nacionales la creación de un centro de interpretación de las islas Chafarinas, proyecto que incluía la r estauración de la iglesia de la Inmaculada Concepción, antigua iglesia del archipiélago.

Este trabajo venía a proponer la investigación, adecuación y transfor mación de dicho templo. Una finca, propiedad del Ministerio de Defensa, para la que se planteó una r ecuperación en varias fases: limpieza, desmontaje y tratamiento del retablo, la reposición del tejado y finalmente la adecuación inter ior del templo a su estado original, restaurándose las pilastras, puertas de madera, coro, y finalmente la distribución del espacio propiamente dicho para su finalidad como centro interpretativo.

El objetivo principal de este proyecto estaba orientado a promocionar nue- 
vas ofertas didácticas y potenciar el conocimiento de este espacio potegido que, a la vez de tratar los temas específicamente medioambientales, podría también exponer los históricos y arqueológicos. Toda esta puesta en valor del lugar hubiera permitido reconocer el valor patrimonial del Refugio y entender el patrimonio como recurso sostenible, así como el fomento de una actitud de ciudadanía activa, receptiva y dialogante ante los pr oblemas de conservación integral. Por otra parte se hubieran pr omocionado los trabajos de in vestigación. Todo ello hubiera sido complementado con la musealización de los yacimientos arqueológicos. Para esta iniciativa, en aquel momento, contamos con la colaboración del Centro de Hijos de Chafarinas, entidad que mantiene viva la herencia cultural de las islas.

\section{Plan Nacional de Acción de Voluntariado del Organismo Autónomo de Par- ques Nacionales}

Durante el verano de 2003 la permanencia en las islas fue constante y las expectativas se multiplicaron gracias a diversas actividades vinculadas a distintas instituciones. El ICM dio un considerab le avance al firmar un nuevo convenio con Parques Nacionales, organizar y coordinar los campos de v oluntariado medioambientales de esta institución, actividad que ya venía desarrollándose en otros Parques del ámbito nacional. De nuevo aumentan las posibilidades de todos aquellos interesados en visitar las islas para colaborar en taras de optimización del medio protegido. Sin embargo, un mayor número de personas iba a acarrear mayores complicaciones. Se inicia entonces un ciclo tr epidante de actividades donde voluntarios, sin límite de edad y procedentes, en su mayoría, de la ciudad de Melilla, participarán en los pr oyectos más recientes enfocados al mantenimiento de las subestructuras y su entomo o a la construcción de instalaciones de apoyo a proyectos biológicos.

En esta nueva fase culminarán propósitos planificados por el ICM y Parques Nacionales. Se acondicionó en varias fases, una zona colindante a la Estación Biológica para construir un vivero destinado a la $\mathrm{r}$ eproducción de especies v egetales autóctonas. Toda el área próxima fue adecuándose en difer entes fases, ampliando las mejoras a todo el rededor del vivero a partir de levantamientos de muros o la restauración de los existentes reutilizando piedras y escombros vertidos en la zona. Para equipar el umbráculo fue necesario instalar depósitos de agua de lluvia a través del reciclado de distintos elementos, conducciones de agua, electricidad, etc. 
Más adelante, este proyecto culminará con la construcción de un vivero de exterior para la fase intermedia de adaptación de las especies plantadas o un pequeño huerto para abastecer en ocasiones a la Estación Biológica.

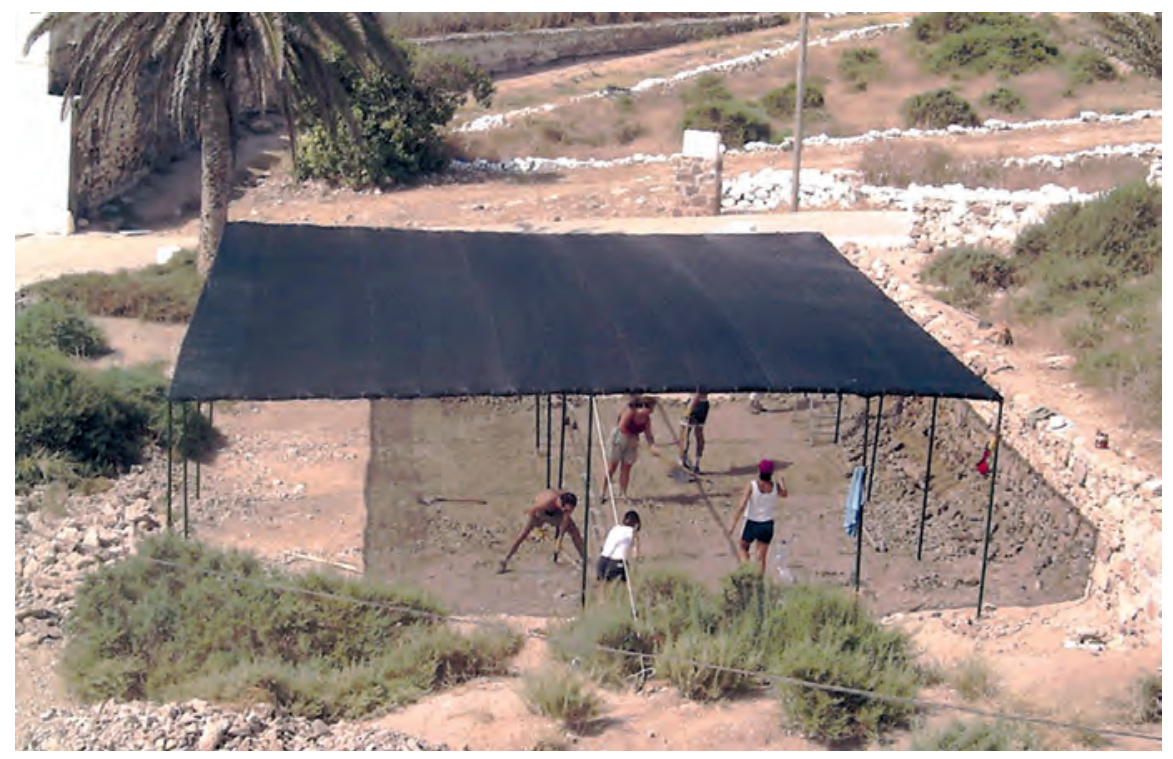

Construcción del vivero junto a la Estación Biológica en el año 2002.

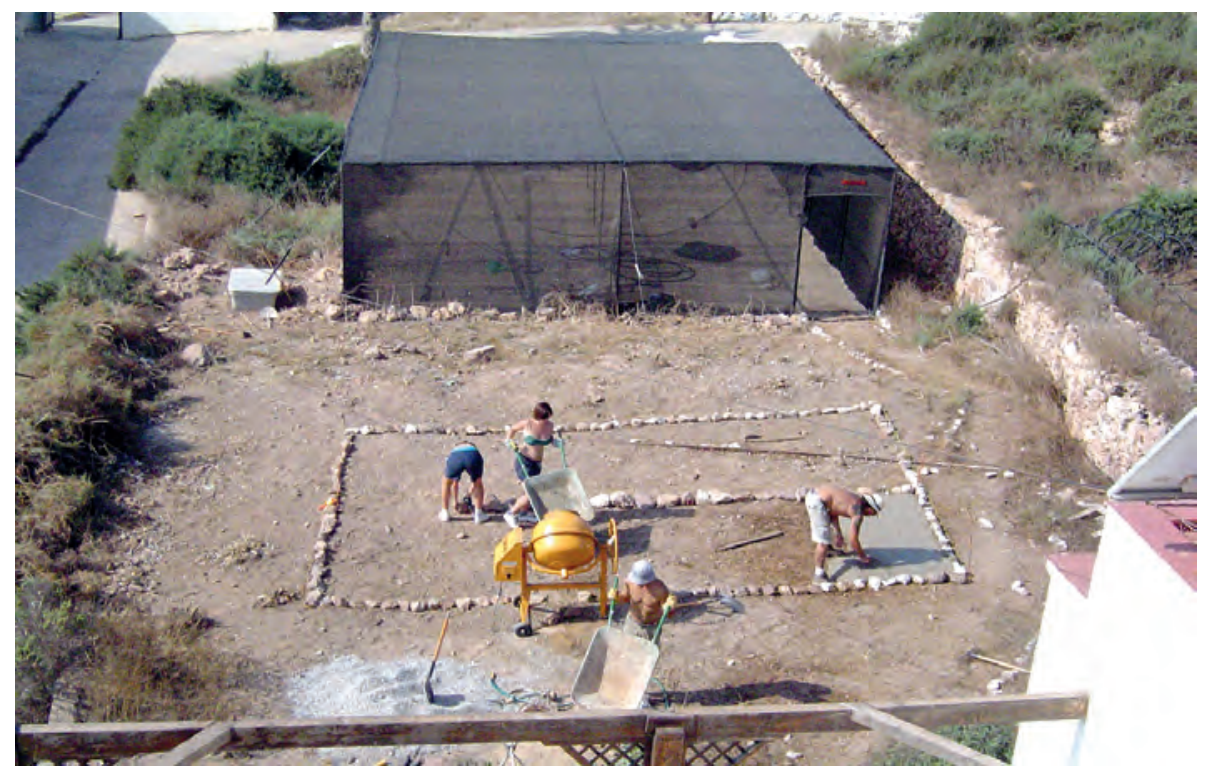

Construcción de un vivero de exterior durante el verano de 2004, junto al umbráculo levantado anterior. 
Igualmente, con la participación de voluntarios se construyó un terrario para el estudio del eslizón pentadáctilo de mediano tamaño, endémico de la franja costera mediterránea magrebí y las islas Chafarinas, concretamente de la isla del Rey, donde es abundante. Así, estos grupos de jóvenes colaboraron de forma indirecta en algunos de los proyectos científicos llevados a cabo por in vestigadores de Parques Nacionales en las islas Otras muchas actividades fueron propuestas y desarrolladas durante los veranos sucesivos, como la fabricación de composteros, levantamientos de muros, mantenimiento y reconstrucción de caminos, actividades que formarían parte de un proyecto común de carácter medioambiental desar rollado por un voluntariado intermitente desplazado a las islas cada verano, siempre dirigidos y coordinados por monitores y personal del ICM.

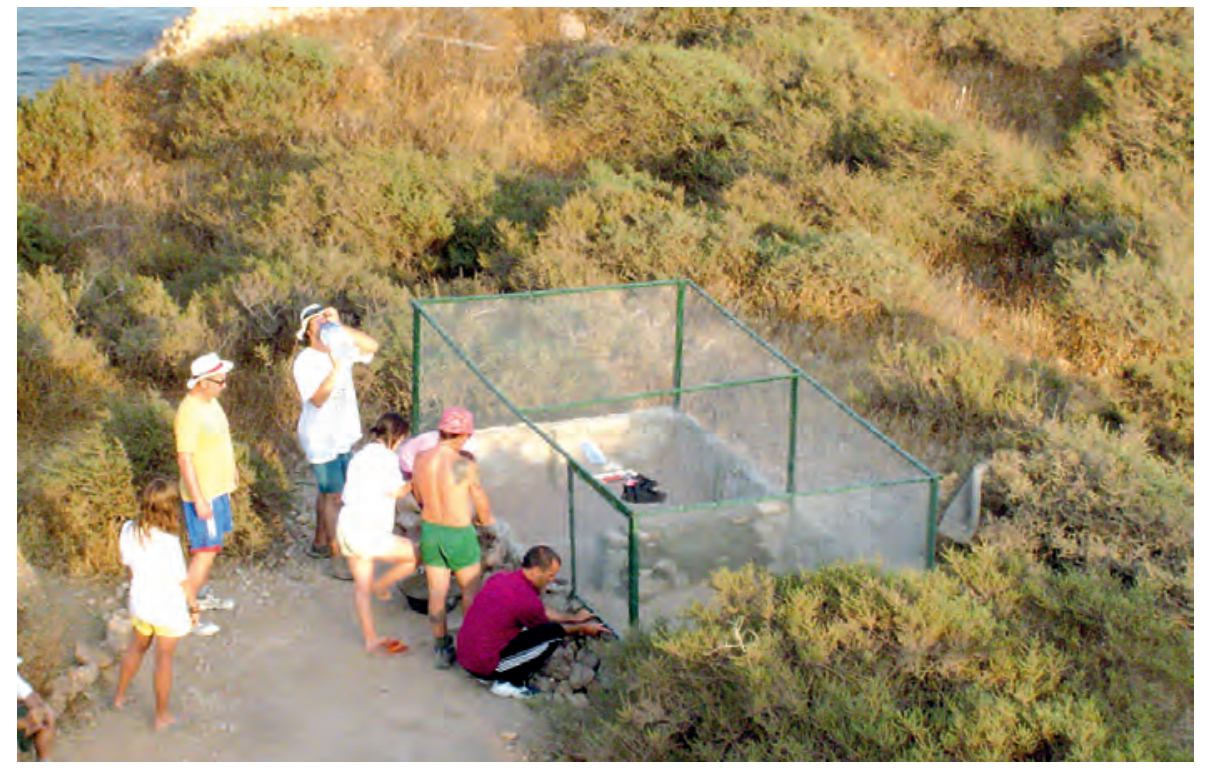

Trabajos durante la construcción de un terrario para el estudio de los eslizones.

\section{EL ICM Y LA CONSEJERÍA DE EDUCA CIÓN. LOS CAMPOS DE VOLUNTARIADO DE JUVENTUD}

Si la arqueología marcó el inicio y caminó paralela a la đolución del ICM, pronto dejaría de ser la única actividad que esta institución desarr ollaría en las islas. Desde el año 2003 los campos de voluntariado de la Consejería de Educa- 
ción y los medioambientales de Parques Nacionales formarían parte importante de la dinámica en el archipiélago.

Los primeros contactos del ICM con el voluntariado de juventud se producirán en 2002, un proyecto que planteará nuevas expectativas de colaboración entre el OAPN y la Consejería de Educación de Melilla. De esta forma, el ICM se encontró gestionando un campo de voluntariado orientado a iniciar a los jórenes participantes en la arqueología. Una opción que va a permitir a un amplio número de civiles residir y disfrutar durante un breve periodo de tiempo en un entorno natural protegido. Un proyecto que comenzó combinando la arqueología teórica en Melilla con las prácticas en las islasSin embargo, este voluntariado será reorientado rápidamente hacia actividades medioambientales desarrolladas íntegramente en la isla de Isabel II. Un convenio con la Consejería de Educación de Melilla permitió que, durante estos años, grupos de jóvenes entre 18 y 30 años de diferentes Comunidades Autónomas pudieran estar en las islas para el desarrollo de actividades relacionadas con el mantenimiento de instalaciones o acciones relacionadas con el medioambiente.

El primer campo de juv entud acogió a un g rupo de universitarios que iniciaría las primeras actividades y per mitió asumir nuevos objetivos. De esta manera, el ICM ampliaba hor izontes y cada año se pr ogramaban multitud de ocupaciones a emprender. Desde aquel momento todo se magnificó, tanto el volumen humano a trasladar, la coordinación de estos grupos o el esfuerzo empleado frente a la administración, igualmente crecerá la responsabilidad y con ella la preocupación de que cada propósito culminara positivamente.

La dinámica de los campos de juventud, originalmente de orientación arqueológica, se fuer on transformando hacia cometidos específicos de carácter medioambiental, aunque en momentos puntuales estos gupos continuarían realizando labores de apoyo al equipo de arqueólogos del ICM.La dinámica de este voluntariado consistía en ejecutar trabajos diseñados a priori entre el ICM y el Organismo Autónomo de Parques Nacionales. Pero también se r eservaba un periodo del día en el que desarrollaban actividades de ocio, todas ellas coordinadas por los monitores con el propósito de fomentar el conocimiento del entorno, la convivencia, el compañerismo y las relaciones tanto entre los propios voluntarios como de estos con el destacamento militar, a los que se les hizo siempre partícipe de las actividades de entretenimiento. Los campamentos se realizaban durante quince días (divididos en dos tumos de una semana), habitualmente 
en el mes de julio. Durante estos años de acción en las islas se distinguier on diferentes proyectos centrados básicamente en dos áreas concretas de la isla de Isabel II, el espacio adyacente a la Estación Biológica, lugar donde viven guardas y biólogos durante el año y, el entorno del faro, lugar donde pernoctaban y convivían los jóvenes voluntarios.

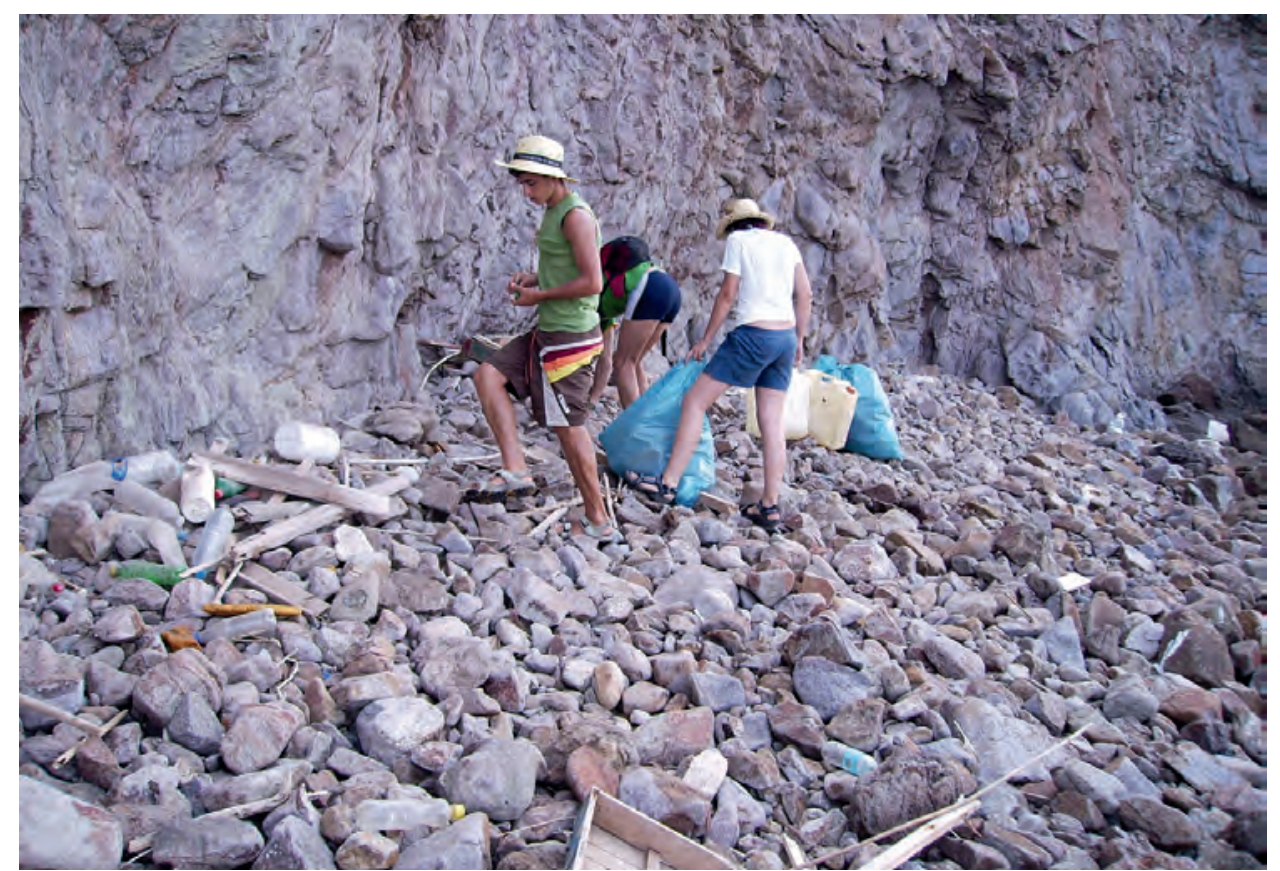

Recogida de residuos de las costas durante los campos de voluntariado en las islas.

El mantenimiento del faro en el extremo norte de la isla de Isabel II conllevó actividades para el sostenimiento de las instalaciones tales como limpieza y pintura. En el entorno de la Estación Biológica, el desescombro y acondicionamiento de zonas destinadas a instalar equipamientos específicos para estudios biológicos fueron las tareas principales. Se reconstruyeron caminos originales desaparecidos y se crearon otros nuevos con técnicas no in vasivas ni agresivas, siempre respetando el entomo y los métodos utilizados en las antiguas construcciones. Los trabajos diarios serían alternados con la limpieza de las costas, recogida de residuos y la consiguiente agrupación de materiales de desecho para su eliminación. En general, este voluntariado sirvió de apoyo en muchos otros proyectos, tanto biológicos como arqueológicos, de tal manera que adelantaron tra- 
bajos antes de la llegada de equipos científicos a a veces, procedieron al acondicionamiento de zonas afectadas por g rupos de investigadores, como por ejemplo, desbrozado y acondicionamiento de zonas para las excaraciones arqueológicas así como cierre de las mismas y repoblación del suelo afectado. A veces, se unieron a campañas de anillamiento de gaviotas o pardelas como apoyo a biólogos desplazados a las islas para tal fin.

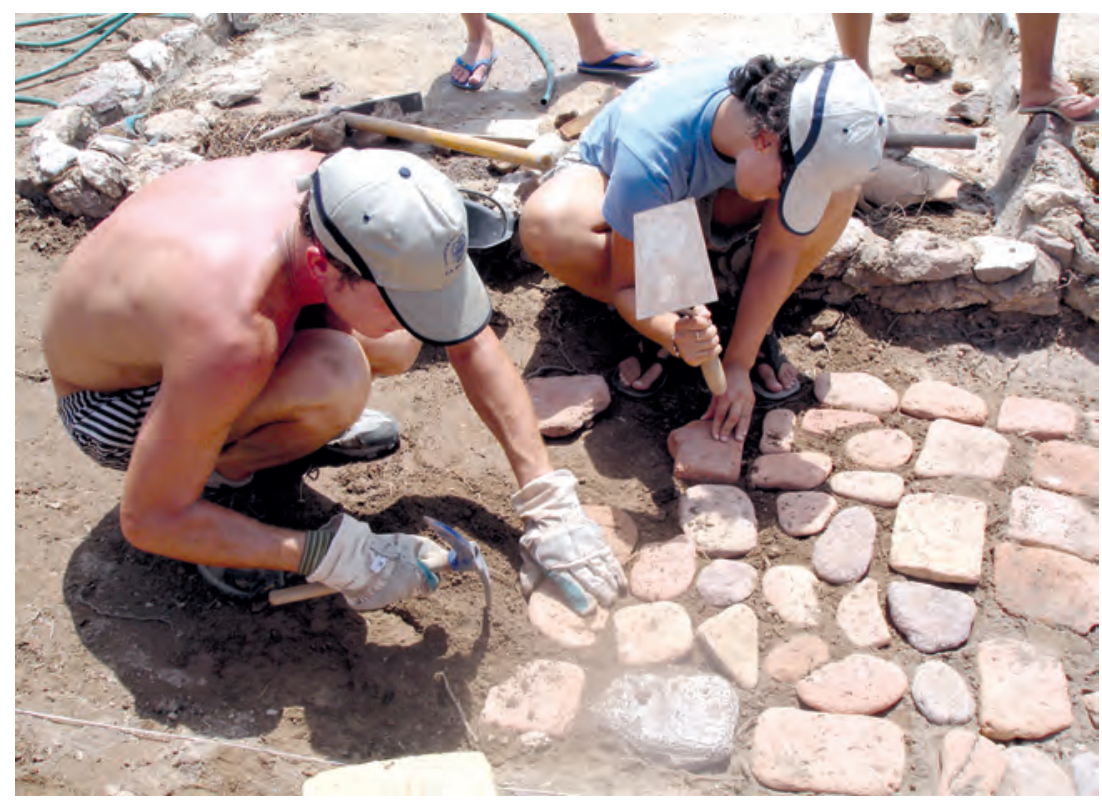

Reconstrucción de calzadas en los alrededores de la Estación Biológica por grupos de voluntarios.

\section{LAS ACTIVIDADES DE PUBLICIDADY LA PUBLICACIÓN DE LAS ACTIVI- DADES}

La divulgación de todas las intervenciones se convertiría en la fase final del proyecto arqueológico anual. Las memorias de excavación de cada una de las campañas y los informes de cada uno de los pryectos realizados, fueron redactados y entregados al Ministerio de Cultura y al OrganismoAutónomo de Parques Nacionales desde el año 2000 al año 2009. En esta documentación se describirían detenidamente todas las actividades de investigación en el año de referencia y permitían ofrecer estos resultados al resto de la comunidad científica. 
Un hito importante en este proceso fue la visita de la entonces ministra de Cultura, Pilar del CastilloVera a la sede del ICM en Melilla en el año 2002 donde pudo apreciar los primeros resultados de las campañas arqueológicas, interesándose por los distintos materiales cerámicos y objetos de piedra que a estaban en fase de estudio.

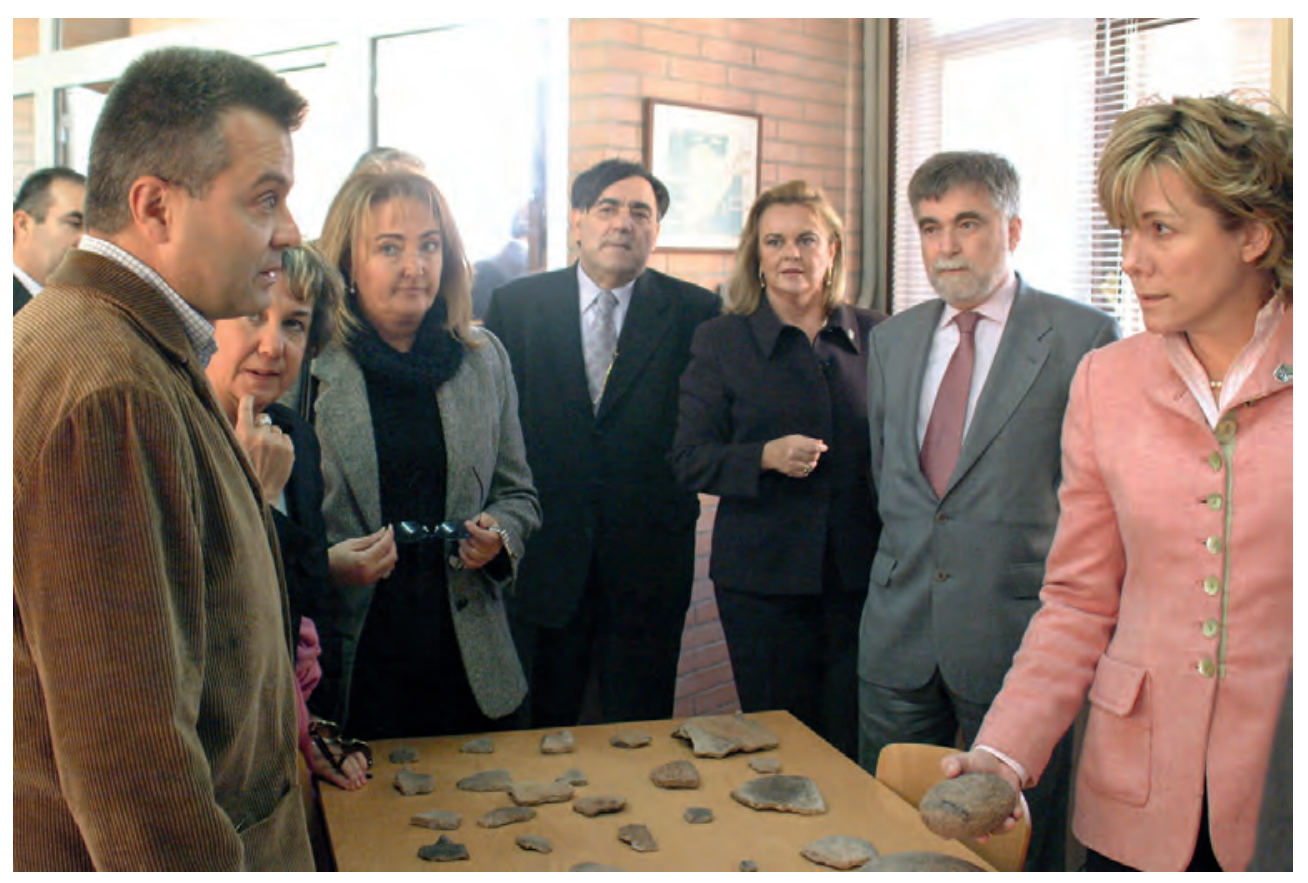

La ministra de cultura Pilar del Castillo en su visita al ICM, apreciando la colección de piezas rescatadas en las primeras campañas. 2002.

El primer aniversario (20 años) del Refug io Nacional de islas Chafar inas fue una de las primeras tareas realizadas. El Organismo Autónomo de Parques Nacionales encargó al ICM una exposición y un ciclo de conferencias que recogieran las actividades que se habían lle vado a cabo en los últimos v einte años desde la creación del Refugio. Las actividades se llevaron a cabo en el Hospital del Rey de Melilla, donde participaron diferentes investigadores y personas vinculadas al trabajo en las islas.

Reconocer la labor investigadora y revisar la dilatada trayectoria científica del profesor Carlos Posac Mon fue otro de los objetivos del ICM. Se organizó un emotivo homenaje al investigador por la gran labor realizada entre los años cin- 
cuenta y, sobre todo, por ser el descubridor de los primeros restos neolíticos en las islas Chafarinas. Aquel acto consistió en una jomada de presentación de todas sus obras compiladas en un único tomo editado por el Instituto de Cultura Mediterránea. Contó con la presencia de Carlos Posac, quien rememoró sus experiencias en las numerosas prospecciones que llevó a cabo en su estancia en Melilla como docente durante los años cuarenta y cincuenta del siglo XX.

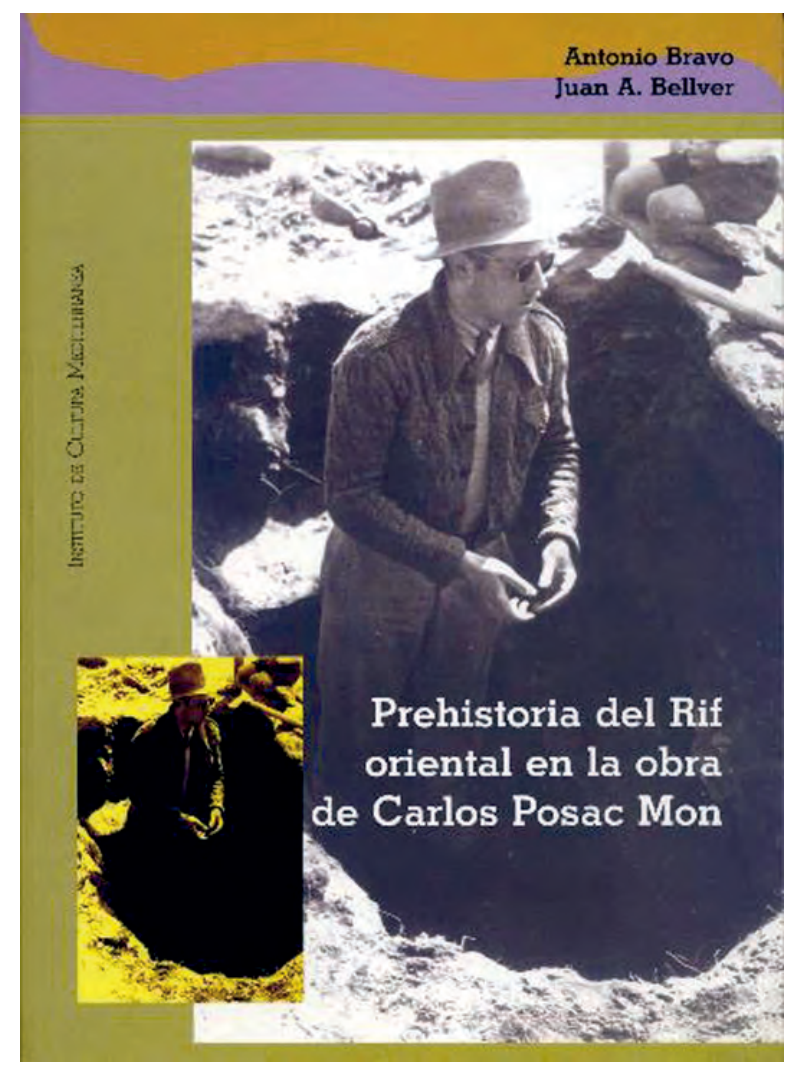

Publicación que rinde homenaje a Carlos Posac Mon.

La exposición en la Fundación GASELEC, Islas Chafarinas, una mirada al pasado, estuvo acompañada de la publicación de las intervenciones arqueológicas en el poblado neolítico de Zafrín hasta el ão 2006. La exposición ofreció de una manera didáctica los resultados y conclusiones más relevantes, materiales obtenidos y principales resultados científicos de este pob lado neolítico cardial. La Fundación GASELEC apoyó este proyecto de investigación casi desde el comien- 
zo, por ello, fue la receptora en el año 2006 de esta importante exposición. Los contenidos y recursos expositivos fueron adaptados a las condiciones del centro de exposiciones de la Fundación. Para ésta se elaboraron recreaciones de numerosas cerámicas halladas en las excaaciones, reproducciones a tamaño natural de las especies más comunes que compartieron la existencia del hombre neolítico en las islas, peces diversos y focas monjes. También se realizó una reproducción de la cabaña que se excavó en la campaña 2004 a tamaño real.
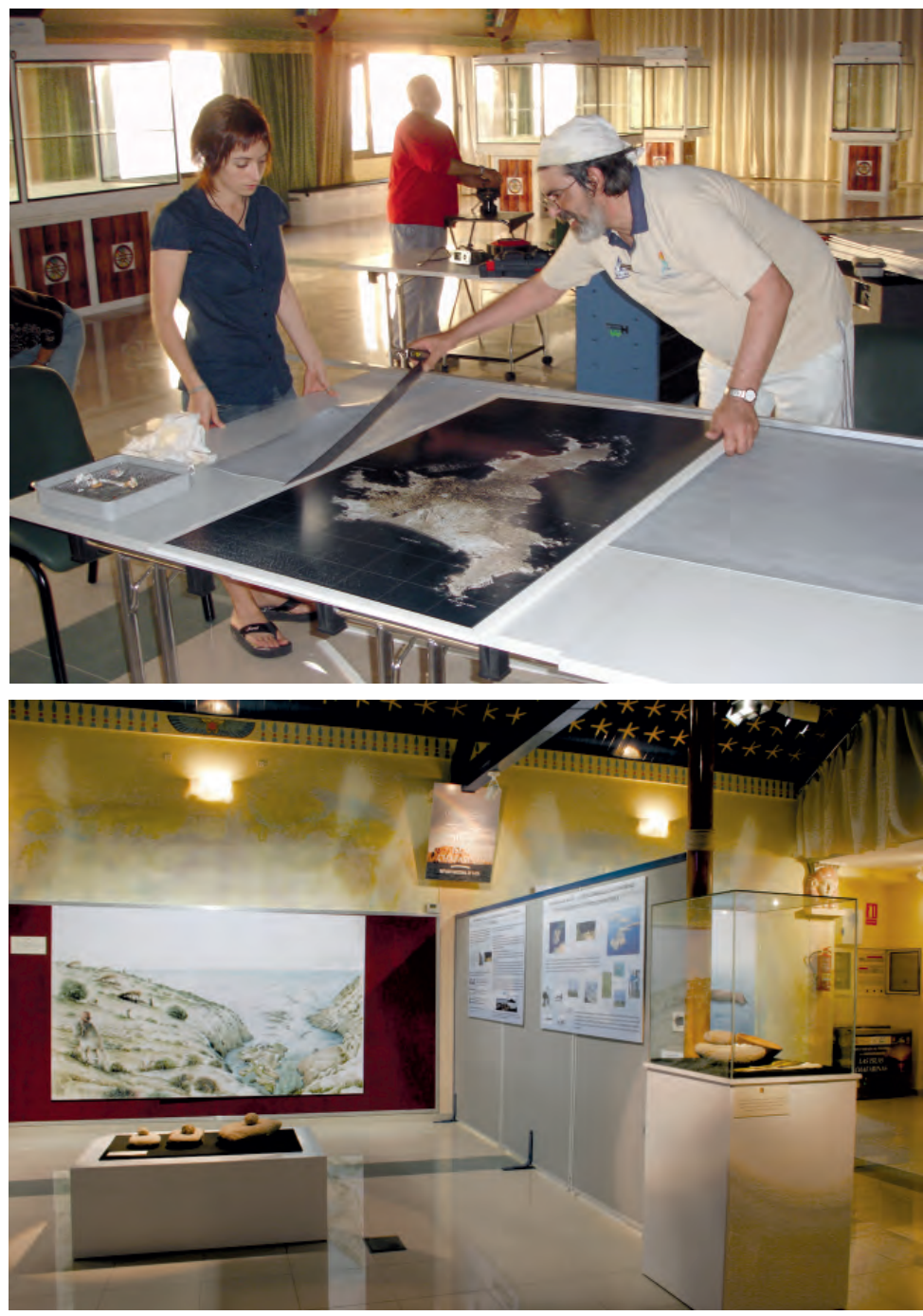

Montaje de la exposición "Una mirada al pasado: Prehistoria de las islas Chafarinas", en la Fundación Gaselec en el año 2006. 
La revista $A k r o s$ ha servido para difundir muchas investigaciones realizadas en Melilla y su entorno más inmediato. Varios números de Akros se han convertido en el sopor te expositivo de las no vedades acontecidas en Chaf arinas. En el año 2003 se pub licó el ar tículo "La estación neolítica al air e libre en las islas Chafarinas. Primera datación radiocarbónica” por J. A. Bellver Garrido y A. Bravo Nieto. En 2005 se publica "El yacimiento neolítico de Zafrín en las islas Chafarinas (Norte de África, España): avance de los r esultados de la campaña de excavación 2004", artículo conjunto de M. Rojo; J. A. Bellver; A. Bravo; R. Garrido; I. García y S. Gámez. En 2007, junto a la exposición en la Fundación GASELEC se publica como catálogo Una mirada al pasado: Prehistoria de las islas Chafarinas. Otras inter venciones arqueológicas también fueron difundidas por Akros como en 2008 con el ar tículo "La Plataforma: un punto estratégico en la isla del Congreso” por S. Gámez Gómez y C. Tejedor Fernández.
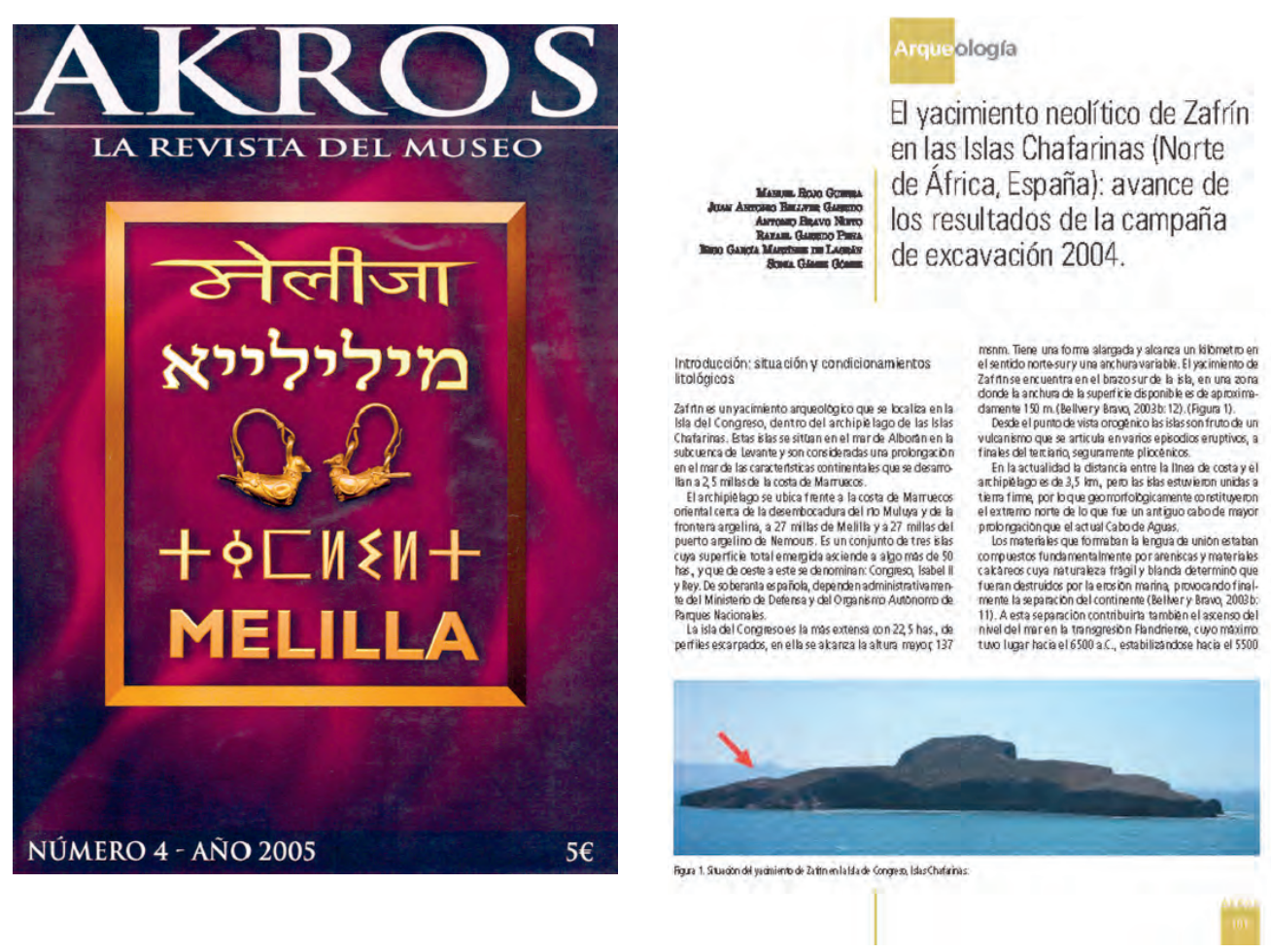

Publicación en la revista del Museo Akros en 2005. 
Otras publicaciones relevantes fueron las actas de la intervención en el IV Congreso de arqueología peninsular en P ortugal en 2004, Do Epipaleolitico ao Calcolítico na Península Ibérica. La ponencia fue "El yacimiento de Zafrín en las islas Chafarinas (Norte de África, España): un nue vo asentamiento del neolítico cardial”, por M. Rojo; J. A. Bellver; A. Bravo; R. Garrido; I. García y S. Gámez. En este Congreso se presentaron los resultados de las tres campañas de excavación desarrolladas en el asentamiento descubierto en la isla del Congreso, en la que se pudieron excavar diversas estructuras de hábitat (hogares, silos, etc.) proporcionando una importante cantidad de materiales arqueológicos de gran interés como cerámicas cardiales, recipientes de cáscara de huevo de avestruz y una industria lítica muy especializada. En la inter vención se definió el y acimiento como un pequeño hábitat con agricultura y ganadería demostradas a tra vés de análisis faunísticos y paleobotánicos, pero con un importante peso de la recolección de moluscos, fechado por C14 a mediados delV milenio cal AC. Estos resultados fueron presentados por el profesor Manuel Rojo Guerra en representación del ICM y de la Universidad de Valladolid.

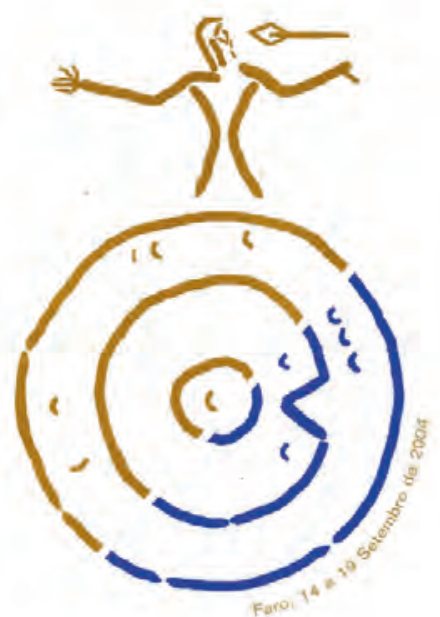

$$
\begin{array}{r}
\text { Do Epipaleolitico ao Calcolitico } \\
\text { na Peninsula Ibérica } \\
\text { actas do ov congresso } \\
\text { de arqueologita peninsular }
\end{array}
$$

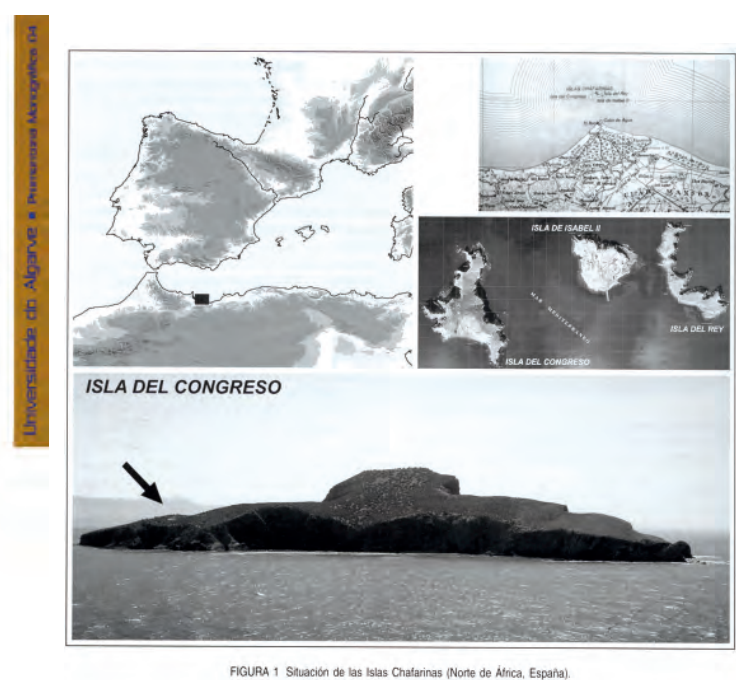

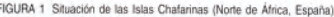

Actas del Congreso de Arqueología peninsular en Portugal en 2004. 
Y finalmente, la obra definitiva que recopila todas las campañas es el libro colectivo editado por la Univ ersidad de Valladolid en el año 2010, Zafrín: un asentamiento del neolítico antiguo en las islas Chaf arinas (Norte de África, España), trabajo para el que se dedicó un tomo de la serie Studia Archaeologica del Departamento de Prehistoria de la Universidad de Valladolid.

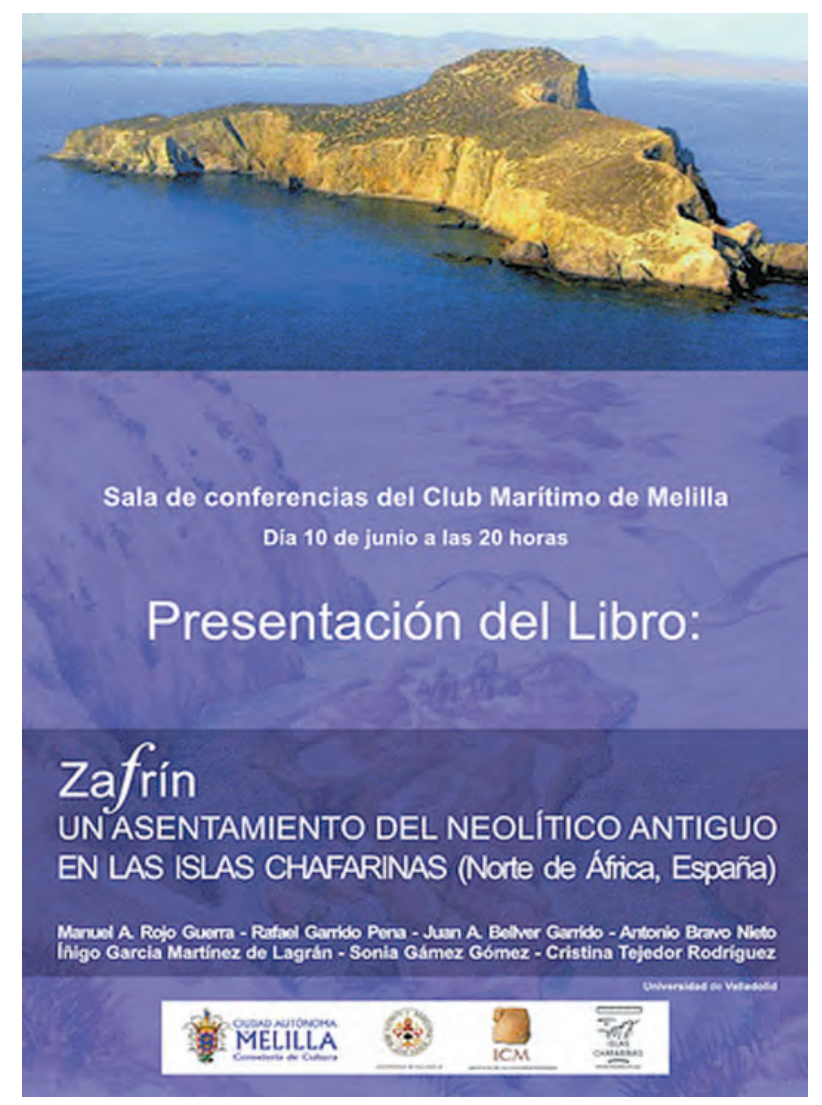

Presentación del libro Zafrín, un asentamiento del Neolítico Antiguo en las islas Chafarinas en el Club Marítimo.

Por otra parte, la revista Ambienta del Ministerio de Medio Ambiente también recogió los inicios de los trabajos de investigación arqueológica en el Refugio Nacional de islas Chafarinas. En 2006 la revista Tierra y Tecnología del ilustre Colegio oficial de Geólogo en Madrid publicó "Islas Chafarinas. La geología de un archipiélago deseado por todos”, escrito por los geólogos José Luís Barrera y Antonio Pineda, activos colaboradores del proyecto de investigación. 


\section{APOYO DE LAS INSTITUCIONES}

El apoyo de las instituciones fue determinante para cada uno de los propósitos que el ICM desar rolló en las islas. Sin este apo yo el Proyecto Chafarinas no habría sido posible, por ello, es de obligado cumplimiento el hacer mención en este apartado de todas aquellas instituciones que de una manera u otra respaldaron las iniciativas que influyeron en la evolución del Instituto de Cultura Mediterránea.

El Organismo Autónomo de Parques Nacionales estuvo interesado y se convirtió en el principal promotor de la investigación desde el mismo año 2001. La Estación Biológica se convirtió durante muchos veranos en el centro base de los trabajos de campo en las islas. Dotado de medios técnicos suficientes, agilizó y permitió el desarrollo del proyecto.

La Comandancia General de Melilla y sus unidades dependientes que custodian las islas: Compañía de Mar, destacamento de Regular es y más recientemente del Tercio de La Legión que apoyaron de diversas maneras a los equipos de trabajo, tanto en el transporte, apoyo sanitario y protección.

La Ciudad Autónoma de Melilla, especialmente la Consejería de Cultura que aportó apoyos necesarios para el tratamiento de los materiales trasladados a Melilla, y su depósito en el museo arqueológico de la Ciudad, donde finalmente forman una sala específica sobre neolítico. La Autoridad Portuaria de Melilla que puso a disposición del Instituto el faro de la isla de Isabel II como dormitorio de los primeros equipos de excavación.

La Fundación GASELEC, ha manifestado un constante apoyo al ICM y sus actividades, abrió sus puertas en 2006 para albergar la exposición Una mirada al pasado: Prehistoria de las islas Chafarinas. También ha colaborado en la edición de varias de sus publicaciones.

Los primeros viajes fueron apoyados por la Salvamar de Melilla, más tarde, el OAPN decide adquir ir una embarcación de ser vicio para las islas que , después de una profunda transformación que incluyó alargar el casco más de un metro y la colocación de dos motores nuevos, serviría de transporte permanente de todos los g rupos de trabajo destinados en las islas y que fue $r$ ebautizada como Zafarín. En algunas ocasiones tuvimos a nuestra disposición los parulleros de la Armada e incluso en más de una ocasión las corbetas que amar raban en el puerto de Melilla, pues la actividad de in vestigación no se extinguió ni tan siquiera durante el conflicto del Perejil. El objetivo científico y cultural de todos los proyectos ha deter minado siempre una colaboración sincera de todos los 
organismos e instituciones que tienen responsabilidad en las islas o que han creído interesante sus objetivos y logros.

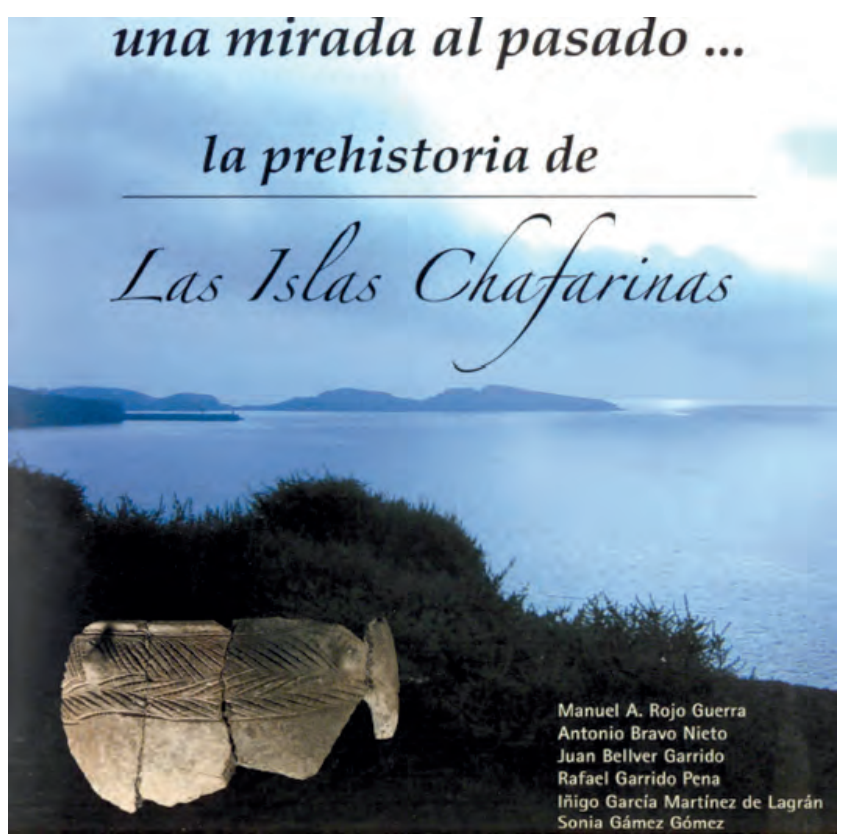

Catálogo de la exposición "Una mirada al pasado... la prehistoria de las islas Chafarinas".

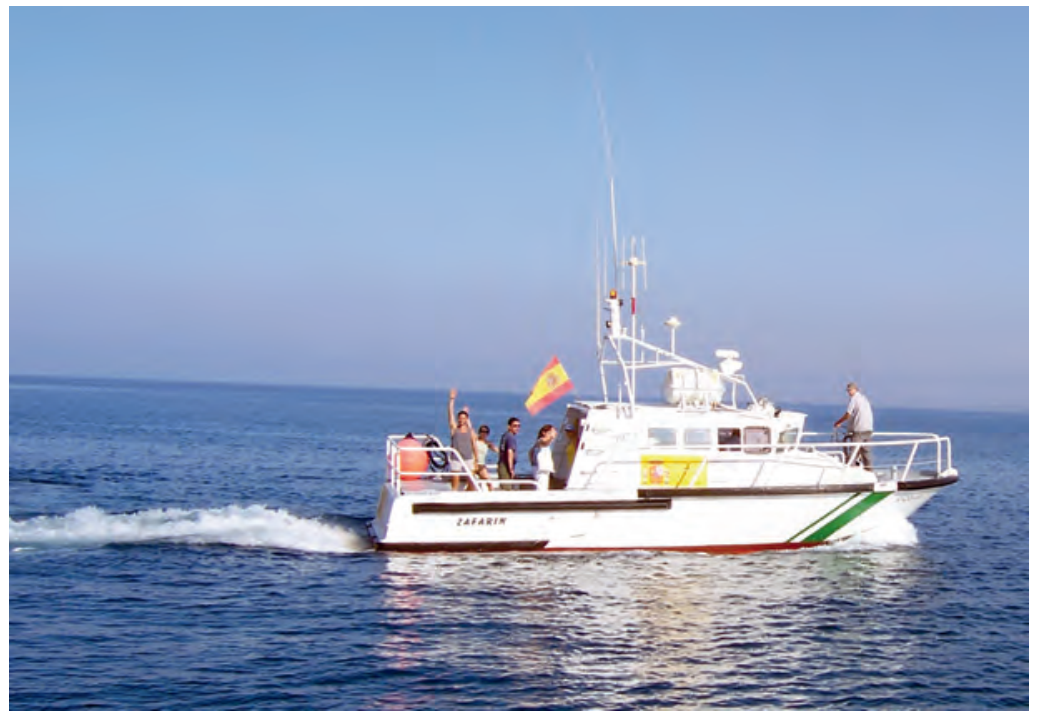

El barco de Parques Nacionales que transporta al personal a las islas fue bautizado con uno de los antiguos nombres de las Chafarinas, Zafarín. 